\section{FRI0748-HPR CROSS-CULTURAL VALIDATION OF THE PORTUGUESE "RHEUMATOID ARTHRITIS IMPACT OF DISEASE" SCORE: CROSS-SECTIONAL STUDY}

R.J.O. Ferreira ${ }^{1,2}$, L. Gossec ${ }^{3}$, S. Hewlett ${ }^{4}$, C. Duarte ${ }^{1,5}$, J.K. Nicklin ${ }^{4}$, J.A.P. da Silva 1,5 , M. Ndosi ${ }^{4} .{ }^{1}$ Centro Hospitalar e Universitário de Coimbra; ${ }^{2}$ UICiSA:E, Escola Superior Enfermagem de Coimbra, Coimbra, Portugal; ${ }^{3}$ Paris 06 University, Paris, France; ${ }^{4}$ University of the West of England, Bristol, United Kingdom; ${ }^{5}$ Faculdade de Medicina da Universidade de Coimbra, Coimbra, Portugal

Background: The Rheumatoid Arthritis Impact of Disease (RAID) score ${ }^{1}$ assesses 7 impact domains of interest for people with rheumatoid arthritis (RA) Its use in research and clinical practice has been growing, and it is already translated into over 70 languages ${ }^{2}$ but the cross-cultural validity of the Portuguese RAID has not been well established.

Objectives: To validate the Portuguese RAID for use in Portugal.

Methods: This was a single centre, cross-sectional validation study involving 2 phases: (i) cognitive debriefing with 38 patients to determine comprehension of the existing ${ }^{2}$ Portuguese RAID (ii) cross-cultural validation using data from adult patients who were willing and able to complete the Portuguese RAID unaided. Analyses included fit to the Rasch model (implying construct validity, reliability and statistical sufficiency), tests for unidimensionality and invariance across different patient subgroups i.e. age, gender, education background, disease duration, function and culture. To test invariance to culture, the Portugal dataset was compared with datasets from France $(n=195)$ and the UK $(n=205){ }^{3}$ RUMM2030 software was used in all analyses.

Results: Phase I led to minor changes in phrasing 3 items to enhance understanding and conceptual equivalence between the original RAID and the Portuguese version. In Phase II, 288 patients were included: mean (SD) age $=60$ (12) years, $82 \%$ females, $76 \%$ with disease duration $\geq 5$ years, $30 \%$ on biologics. The Portuguese RAID was shown to have adequate fit to the Rasch model and high internal consistency (Table 1). Unidimensionality and invariance to age, gender, disease duration and function were confirmed (data not shown). The scale was well targeted for patients with different levels of disease impact (Figure 1). Pooling the datasets from Portugal, France and the UK revealed no cultural response bias (Table 1). RAID was then calibrated into logit-based scores to enable parametric analyses and bias-free cross-cultural comparisons if desired (data not shown).

Table 1. Results of Rasch analysis from pooled data

\begin{tabular}{|c|c|c|c|c|c|c|c|}
\hline \multirow[t]{2}{*}{ Country } & \multirow[t]{2}{*}{$\mathrm{N}$} & \multirow[t]{2}{*}{$\begin{array}{c}\text { RAID } \\
\text { (n items) }\end{array}$} & \multicolumn{2}{|c|}{$\begin{array}{l}\text { Fit Residual } \\
\text { Mean (SD) }\end{array}$} & \multicolumn{2}{|c|}{$\begin{array}{c}\text { Chi }^{2} \\
\text { Interaction }\end{array}$} & \multirow[t]{2}{*}{$\begin{array}{c}\text { Person Separation } \\
\text { Index }\end{array}$} \\
\hline & & & Item & Person & Value (DF) & $\overline{p \text {-value }}$ & \\
\hline Portugal & 288 & 7 & $-0.13(2.53)$ & $-0.67(1.60)$ & $40.50(35)$ & 0.24 & 0.94 \\
\hline UK & 205 & 7 & $0.22(1.72)$ & $-0.44(1.37)$ & $40.50(35)$ & 0.17 & 0.93 \\
\hline France & 195 & 7 & 0.19 (1.99) & $-0.71(1.57)$ & $25.69(21)$ & 0.22 & 0.91 \\
\hline \multirow[t]{2}{*}{ Pooled } & 688 & 7 & $-0.06(3.48)$ & $-0.65(1.55)$ & $94.88(63)$ & 0.01 & 0.93 \\
\hline & & $6^{\star}$ & $-0.34(3.88)$ & $-0.63(1.44)$ & $66.04(54)$ & 0.13 & 0.93 \\
\hline \multicolumn{4}{|c|}{ Expected values for perfect fiD (1) } & $0(1)$ & & $>0.05$ & $>0.85$ \\
\hline
\end{tabular}

$\mathrm{DF}$, degrees of freedom; * 6 items for cross-cultural comparisons (items 2 "Function" and 5 "Physical well-being" combined).

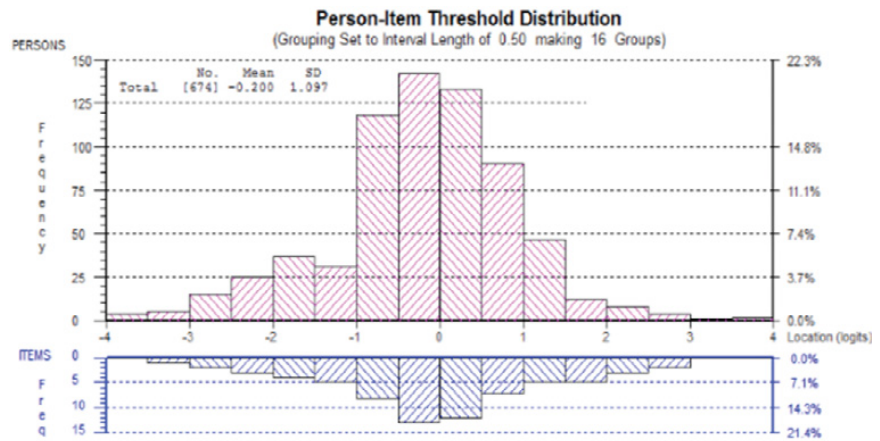

Figure 1. Distribution of items and persons along the same scale (logit score) confirming good targeting of the RAID.

The $x$-axis is the logit score and represents the interval scaling of the items according to the Rasch model, with -4 being no impact and 4 being high impact of disease.

Conclusions: This study confirms the Portuguese RAID as a robust unidimensional tool for use in Portugal. The raw scores of the 7-item RAID can be used with confidence in clinical practice. Conversion charts are available to enable accurate cross-cultural comparisons across Portugal, France and the UK.

\section{References:}

[1] Gossec L, et al. Ann Rheum Dis, 2011;70:935-42.

[2] EULAR RAID and PsAID Questionnaires. Available from http://www.eular.org/ tools_products_.cfm Accessed 25th Jan. 2017

[3] Hewlett S, et al. Ann Rheum Dis, 2015;74:Suppl 2:559.

Disclosure of Interest: None declared

DOI: 10.1136/annrheumdis-2017-eular.2754

\section{FRI0749-HPR THE EFFECT OF A SUPPORTED OSTEOARTHRITIS SELF-MANAGEMENT PROGRAM ON PHYSICAL ACTIVITY, PAIN, QUALITY OF LIFE AND SELF-EFFICACY AN INTERVENTION STUDY WITH A REFERENCE GROUP}

T.S.J. Jönsson ${ }^{1}$, C.A. Thorstensson ${ }^{2}$, E. Ekvall Hansson ${ }^{3}$, L. Dahlberg ${ }^{4}$. ${ }^{1}$ Department of orthopaedics, Institute of Clinical Sciences, Lund; ${ }^{2}$ Department of Clinical Neuroscience and Rehabilitation, Institute of Neuroscience and Physiology, Gothenburg; ${ }^{3}$ Department of Health Sciences, Division of Physiotherapy; ${ }^{4}$ Department of Orthopedics, Institute of Clinical Sciences, Lund, Sweden

Background: People with osteoarthritis are less physical active than others and a large part are sedentary. It is unclear how to increase the physical activity level for patients with osteoarthritis.

Objectives: The primary aim of this study was to evaluate the effects from an evidence based supported osteoarthritis self-management program in patients with knee or hip osteoarthritis on physical activity. Secondary aim were to evaluate the effect on pain, quality of life and self-efficacy.

Methods: An intervention study with a reference group in which 104 patients (29-75 years) with knee $(n=84)$ or hip $(n=20)$ osteoarthritis participated in the intervention. Patients were referred to a supported osteoarthritis selfmanagement program that include physical therapist delivered information and individually adapted exercise. The reference group comprised 28 patients (49-75 years) with knee $(n=16)$ or hip $(n=12)$ osteoarthritis from the waiting list at an orthopedic university clinic. Outcome measurements were made at baseline, 3 and 12 months. Physical activity were objective measured with an accelerometer. The secondary outcomes were patient reported, Visual Analog Scale (VAS) were used to measure pain, EQ-5D to measure quality of life and Arthritis Self Efficacy Scale to measure self-efficacy.

Results: The supported osteoarthritis self-management program did not improve physical activity $(p=0,77)$ between baseline and 3 month follow up compared to the reference group. But it did improve pain $(p=0,02)$, quality of life $(p=0,002)$, self-efficacy-other $(p=0,015)$ and self-efficacy-pain $(p=0,033)$ between baseline and 3 month follow up compared to the reference group. The improvements in pain and quality of life in the intervention group persisted at 12 month follow-up. Conclusions: The supported osteoarthritis self-management program delivering information and individualized exercise according to the national program BOA in Sweden improved pain, quality of life and self-efficacy. Despite these results, the physical activity level were not improved. More research on how to improve the physical activity in patients with osteoarthritis is needed.

\section{References:}

[1] Cross M, Smith E, Hoy D, Nolte S, Ackerman I, Fransen M, et al. The global burden of hip and knee osteoarthritis: estimates from the global burden of disease 2010 study. Ann Rheum Dis. 2014;73(7):1323-30.

[2] Zhang W, Nuki G, Moskowitz RW, Abramson S, Altman RD, Arden NK, et al. OARSI recommendations for the management of hip and knee osteoarthritis: part III: Changes in evidence following systematic cumulative update of research published through January 2009. Osteoarthritis Cartilage. 2010; 18(4):476-99.

[3] Thorstensson CA, Garellick G, Rystedt H, Dahlberg LE. Better Management of Patients with Osteoarthritis: Development and Nationwide Implementation of an Evidence-Based Supported Osteoarthritis Self-Management Programme. Musculoskeletal care. 2014

[4] Wallis JA, Webster KE, Levinger P, Taylor NF. What proportion of people with hip and knee osteoarthritis meet physical activity guidelines? A systematic review and meta-analysis. Osteoarthritis Cartilage. 2013;21(11):1648-59.

Acknowledgements: Grants were received from The Academy of Caring Sciences, Skåne University Hospital and The Swedish Rheumatism Association in Gothenburg.

Disclosure of Interest: None declared

DOI: 10.1136/annrheumdis-2017-eular.5711

\section{FRI0750-HPR CONSTRUCT VALIDITY OF THE INTERNATIONAL PHYSICAL ACTIVITY QUESTIONNAIRE - LONG FORM IN} PATIENTS WITH AXIAL SPONDYLOARTHRITIS

T.W. Swinnen $^{1,2,3}$, S. Willemijns ${ }^{2}$, W. Dankaerts ${ }^{1}$, R. Westhovens ${ }^{2,3}$, K. de Vlam ${ }^{2,3} .{ }^{1}$ Research Group for Musculoskeletal Rehabilitation; ${ }^{2}$ Skeletal Biology and Engineering Research Center, KU Leuven; ${ }^{3}$ Division of Rheumatology, University Hospitals Leuven, Leuven, Belgium

Background: Physical activity (PA) is increasingly recognized as an important outcome measure in patients with axial spondyloarthritis (axSpA). Indeed, PA interventions in axSpA have shown to improve clinical status and to reduce comorbidity. The International Physical Activity Questionnaire-Long Form (IPAQLF) may be a feasible self-reported PA measurement tool, but its validity is unknown in axSpA.

Objectives: To establish the convergent construct validity of the IPAQ-LF in axSpA Methods: Forty patients with axSpA (Male/Female: 24/16; Mean $\pm S D$, Age: $44.38 \pm 11.30$ yrs, BMI: $26.27 \pm 5.11 \mathrm{~kg} / \mathrm{m}^{2}$, disease duration: $11.40 \pm 9.50$ yrs, disease activity (BASDAI): $3.69 \pm 2.59$ ) completed the IPAQ-LF and wore the SenseWear Pro3 Multisensor Armband during five consecutive days (three weekdays and two weekend days). A priori, significant directional associations 